\title{
Global Development of Children with Intellectual Disability: Intrinsic Factors versus Extrinsic Interventions
}

\author{
Fuzhou Wang
}

Group of Neuropharmacology and Neurophysiology, Division of Neuroscience, The Bonoi Academy of Science and Education, Chapel Hill, NC 27510, USA.

\footnotetext{
"In special education, there's too much emphasis placed on the deficit and not enough on the strength." -Temple Grandin
}

\begin{abstract}
How to realize the global development of children with intellectual disability is a complicated social issue. When a child has intellectual disability or is on the verge of intellectual disability, the environment the child is in and the humanistic support the child obtains play a vital role in achieving the global development. Given the intrinsic factors cannot be changed, and then the extrinsic interventions subsequently become the only means for children with intellectual disability to achieve personal development. However, conventional education cannot meet the special needs of children with intellectual disabilities, so training special supporting teachers has become the best choice to achieve this goal.
\end{abstract}

Keywords: Intellectual Disability, Education, Extrinsic Intervention, Intrinsic Factor, Equity

$I^{1}$

NDIVIDUAL global development is an extremely complicated process involving intrinsic and extrinsic factors function well mutually. Deficiency in any one the contributing factors would result in developmental retardation, of which intellectual disability (ID) is one of the focal dimensions we care of (Marrus \& Hall, 2017; Shea, 2012). In comparison, the intrinsic factors of ID generally refer to the inheritance characteristics that individuals do not have

(C) 2021 Insights Publisher. All rights reserved.

(c) (i) (8) Creative Commons Non Commercial CC BY-NC: This article is distributed under the terms of the Creative Commons Attribution-NonCommercial 4.0 License

(http://www.creativecommons.org/licenses/by-nc/4.0/) which permits non-commercial use, reproduction and distribution of the work without further permission provided the original work is attributed by the Insights Publisher. 
choices to change them. As thus, for an individual with the medically diagnostic inheritance features of ID, the only hope for reaching a relatively better global development level is extrinsic interventions (De Giorgio, 2017). Therefore, we need to provide them with individualized optimal interventions delivered by well-trained supporting personnel. However, training the supporting team who possesses specific properties to identify and deliver the needed interventions is a systemic project (Gaggioli \& Sannipoli, 2021).

\section{Intrinsic and Extrinsic Factors of Intellectual Disability}

As indicated by a meta-analysis of population-based studies, the prevalence of ID across the world was 10.37/1,000 population (Maulik et al., 2011). Of them, approximately 17\%-47\% had exogenous and genetic causes (Printzlau et al., 2017; Webb et al., 1987). According to the World Health Organization (WHO), "ID is a significantly reduced ability to understand new or complex information and to learn and apply new skills (impaired intelligence). This results in a reduced ability to cope independently (impaired social functioning), and begins before adulthood, with a lasting effect on development." (World Health Organization, 2021). Based on this definition with the combined indication of the American Association on Intellectual and Developmental Disabilities (AAIDD) (2021), the concomitant limitations of ID should be considered simultaneously (Table 1).

Medically, the intrinsic factors of ID are generally indicating those that cannot be changed with exogenous interventional maneuvers, such as genetic and hereditary factors. As listed in the Table 2, these intrinsic factors are either from the chromosomal or hereditary disorders. Meanwhile, some acquired factors like congenital and developmental elements are also considered as the partially modifiable factors if the preventive procedures were given promptly. However, if these early preventive interventions were not provided, then the ID resulted from them was also regarded as the nonchangeable intrinsic factors (Table 2).

The development of each individual is not only based on good innate factors, but the environmental and sociocultural factors also play an irreplaceable role (Table 2). These extrinsic contributing factors are relatively much easier to be adjusted if adequate attention was paid to. Although these factors are strongly associated with changes in society, economy, culture, education, psychology, family, and health, the proportion of each component that can be intervened is very large. As demonstrated by Gaggioli \& Sannipoli (2021) that training supporting teachers for ID students possesses particular importance in helping the individual student as well as the whole community.

\section{Extrinsic Intervention of Intellectual Disability}




\section{Table 1. Limitations of Intellectual Disability.}

Communication

\begin{tabular}{l}
\hline Personal care \\
\hline Home life \\
\hline Social skills \\
\hline Utilization of the community \\
\hline Self-governance \\
\hline Health and safety \\
\hline Functional academic skills \\
\hline Leisure time \\
\hline Work \\
\hline Note: Revised from the American Association on Intellectual and Developmental Disabilities \\
(AAIDD).
\end{tabular}

Since we cannot change the intrinsic factors of ID, the only thing we can rely on is the extrinsic interventions (De Giorgio, 2017). In a particular society, individuals still cannot easily change their social, economic, cultural, and health care environment. Therefore, for the global development of ID individuals, it can only be counted on to seek appropriate educational interventions. In this process, education shoulders an inestimable task. However, conventional education cannot really satisfy these ID children to achieve breakthrough development, which can only be achieved through special education delivered by educators who received special training.

The emergence of special education provides hope for the development of ID children, but it also in turn puts forward higher requirements for those engaged in special education work. Special education workers need to know the skills and methods of conventional education, but also need to understand the special needs of each ID child, so as to guide and educate them with their own knowledge and judgment. Therefore, the training of special supporting teachers has become an essential project.

As mentioned by Gaggioli \& Sannipoli (2021), teachers who receive special supporting training must clearly know the attitude theory of the threefactor model and corresponding five dimensions it contains, through which these teachers would these teachers will get vocational training, teaching experience, perception of effectiveness in their own knowledge regarding ID, perceived support, and promotion of positive attitudes towards ID. There is no doubt that such special supporting training of ID teaching personnel will produce a great positive impact on the global development of ID children. Of course, for every ID individual, the realization of its global development requires a multidisciplinary comprehensive intervention that includes medicine (family, pediatrics, neurology, and psychiatry), psychology (clinical and educational), education (conventional and special), rehabilitation (physical, occupational, and recreational), nursing, and social work (Katz \& LazcanoPonce, 2008; Matson \& Shoemaker, 2011). 
Table 2. Intrinsic and Extrinsic Factors of Intellectual Disability.

\begin{tabular}{l}
\hline Intrinsic Factors \\
\hline Down Syndrome \\
\hline Fragile X Chromosome Syndrome \\
\hline Prader-Willi Syndrome \\
\hline Rett Syndrome \\
\hline Neurofibromatosis \\
\hline Tuberous Sclerosis \\
\hline Lesch-Nyhan Syndrome \\
\hline Adrenoleukodystrophy \\
\hline Phenylketonuria \\
\hline Mowat-Wilson Syndrome \\
\hline Galactosemy \\
\hline Tay-Sachs Disease \\
\hline Glycogen Deposit Disease \\
\hline Partially Intrinsic Factors \\
\hline Neonatal Hypothyroidism \\
\hline Lead Poisoning \\
\hline Fetal Alcohol Syndrome \\
\hline Prenatal Exposure To Substances \\
\hline Rubella \\
\hline Cytomegalic Inclusion Body Disease \\
\hline Syphilis \\
\hline Toxoplasmosis \\
\hline Simple Herpes (Genital Type II) \\
\hline Prenatal Factors: Toxemia; Uncontrolled Diabetes; Intrauterine Malnutrition; Vaginal \\
\hline Hemorrhages; Placenta Previa; Umbilical Cord Prolapse \\
\hline Perinatal Period: Prolonged fetal suffering with neonatal anoxia; Asphyxia related with \\
suffocation; Inadequate application of high forceps or a poorly applied Kristeller \\
maneuver \\
\hline Postnatal Period: Encephalopathy from hyperbilirubinemia (kernicterus); Encephalic \\
traumatism; Infections (encephalitis and meningitis) \\
\hline Extrinsic Factors \\
\hline Society: Underdevelopment \\
\hline Economy: Poverty \\
\hline Culture: Backwardness \\
\hline Education: Low-level stimulation \\
\hline Psychology: Distortion \\
\hline Family: Divorce or Instability \\
\hline Health: Scarcity or Inadequate \\
\hline Note: Revised from Katz \& Lazcano-Ponce, 2008. \\
\hline
\end{tabular}

\section{Concluding Remarks}


True educational equity is not only reflected in each child's access to educational opportunities, but also in whether those ID children are given sufficient attention and spare no effort to promote their global development. The global development of ID children requires the contribution of special teachers, which requires special training programs to provide sufficient professional training for these supporting teachers. The intrinsic factors that lead to a child's ID cannot be changed, whereas we can provide the possibility for its global development, at least in part, with the help of strong extrinsic educational interventions delivered by supporting teachers who received special training.

\section{References}

American Association on Intellectual and Developmental Disabilities. (2021). Intellectual disability. Last access at: April 17, 2021. Retrieved from:

https://www.aaidd.org/intellectual-disability

De Giorgio, A. (2017). The roles of motor activity and environmental enrichment in intellectual disability. Somatosensory \& Motor Research, 34(1):34-43. DOI: https://doi.org/10.1080/08990220.2016.1278204

Gaggioli, C., \& Sannipoli, M. (2021). Improving the training of support teachers in Italy: The results of a research on attitudes aimed at students with Intellectual Disabilities. Science Insights Education Frontiers, 8(2):1037-1057. DOI: https://doi.org/10.15354/sief.21.or021

Katz, G., \& Lazcano-Ponce, E. (2008). Intellectual disability: definition, etiological factors, classification, diagnosis, treatment and prognosis. Salud Publica de Mexico, 50 Suppl 2:S132-S141. DOI: https://doi.org/10.1590/s0036-36342008000800005

Marrus, N., \& Hall, L. (2017). Intellectual disability and language disorder. Child and Adolescent Psychiatric Clinics of North America, 26(3):539-554. DOI: https://doi.org/10.1016/j.chc.2017.03.001

Matson, J.L., \& Shoemaker, M.E. (2011). Psychopathology and intellectual disability. Current Opinion in Psychiatry, 24(5):367-371. DOI: https://doi.org/10.1097/YCO.0b013e3283422424

Maulik, P.K., Mascarenhas, M.N., Mathers, C.D., Dua, T., \& Saxena, S. (2011). Prevalence of intellectual disability: A meta-analysis of population-based studies. Research in Developmental Disabilities, 32(2):419-436. DOI: https://doi.org/10.1016/j.ridd.2010.12.018

Printzlau, F., Wolstencroft, J., \& Skuse, D.H. (2017). Cognitive, behavioral, and neural consequences of sex chromosome aneuploidy. Journal of Neuroscience Research, 95(1-2):311-319. DOI: https://doi.org/10.1002/jnr.23951

Shea S.E. (2012). Intellectual disability (mental retardation). Pediatrics in Review, 33(3):110-121. DOI: https://doi.org/10.1542/pir.33-3-110

Webb, T.P., Thake, A.I., Bundey, S.E., \& Todd, J. (1987). A cytogenetic survey of a mentally retarded school-age population with special reference to fragile sites. Journal of Mental Deficiency Research, 31(Pt 1):61-71. DOI: https://doi.org/10.1111/j.1365-2788.1987.tb01342.x 
World Health Organization. (2021). Definition: Intellectual disability. Last access at: April 17, 2021. Retrieved from: https://www.euro.who.int/en/healthtopics/noncommunicable-diseases/mental-health/news/news/2010/15/childrensright-to-family-life/definition-intellectual-disability

Correspondence to: Fuzhou Wang, M.D., Ph.D. Group of Neuropharmacology and Neurophysiology Division of Neuroscience The Bonoi Academy of Science and Education,

Chapel Hill, NC 27510

USA.

Email:fred.wang@basehq.org

Conflict of Interests: None.

Doi: 10.15354/sief.21.co005 